\title{
Evaluation of the tensile properties of a material through spherical indentation: definition of an average representative strain and a confidence domain
}

\author{
Charbel Moussa ${ }^{* 1,3}$, Xavier Hernot ${ }^{1,2}$, Olivier Bartier ${ }^{1,2}$, Guillaume \\ Delattre $^{3}$, Gérard Mauvoisin ${ }^{1,2}$ \\ 1 LGCGM EA3913, Université de Rennes1-INSA de Rennes, 20 Avenue des Buttes de Coësmes, 35708 Rennes \\ Cedex 7, France \\ 2 IUT de Rennes 1, 3 rue du Clos Courtel, 35704 Rennes Cedex, France. \\ 3 Faurecia Automotive Seating, Le Pont de Vère, 61100 Caligny, France \\ Corresponding author: Charbel Moussa, Tel: +332 23232031 E-mail address: \\ charbel.moussa@univ-rennes1.fr.
}

\begin{abstract}
In the present article, a new method for the determination of the hardening law using the load displacement curve, $F-h$, of a spherical indentation test is developed. This method is based on the study of the error between an experimental indentation curve and a number of finite elements simulation curves. For the smaller values of these errors, the error distribution shape is a valley, which is defined with an analytic equation. Except for the fact that the identified hardening law is a Hollomon type, no assumption was made for the proposed identification method. A new representative strain of the spherical indentation, called "average representative strain", $\varepsilon_{a R}$ was defined in the proposed article. In the bottom of the valley, all the stress-strain curves that intersect at a point of abscissa $\varepsilon_{a R}$ lead to very similar indentation curves. Thus, the average representative strain indicates the part of the hardening law that is the better identified from spherical indentation test. The results show that a unique material parameter set (yield stress $\sigma_{y}$, strain hardening exponent $n$ ) is identified when using a single spherical indentation curve. However, for the experimental cases, the experimental imprecision and the material heterogeneity lead to different indentation curves, which makes the uniqueness of solution impossible. Therefore, the identified solution is not a single curve but a domain that is called "solution domain" in the yield stress-work hardening exponent diagram, and "confidence domain" in the stress-strain diagram. The confidence domain gives clear answers to the question of uniqueness of the solution and on the sensitivity of the indentation test to the identified hardening laws parameters.
\end{abstract}

\section{1-Introduction}

The indentation test is widely used for the determination of the stress-strain curve of materials [1-22]. One of the advantages of this technique is that it is nondestructive and can be applied to materials in some conditions for which the classic tensile test cannot be done. For example, the indentation test can be applied both to small material samples and to plastically graded materials. One of the disadvantages of the indentation test is that the field of strain in the deformed sample is not homogenous which makes it difficult to identify the hardening law of the material. The application of the concept of the representative strain can significantly simplify the analysis of the indentation response and has often been used in the stress-strain curve determination from the indentation test [1-19]. In 1908, Meyer [23] established a relationship between the mean pressure of the material under spherical indentation and the contact radius over indenter radius $(a / R)$ ratio. For a given $a / R$ ratio, the mean pressure should 
be the same using different radius of spherical indentation. Based on this observation, Meyer related the angle of indentation to the $a / R$ ratio [22]. The concept of the representative strain was later introduced by Tabor for Vickers and spherical indentation [1]. Tabor proposed a relationship between the $a / R$ ratio and the representative strain. In the case of spherical indentation, the representative strain depends on the penetration depth of the indenter into the material, $h,[1-14]$ or the contact radius, $a,[1,8-19]$. In this study, only the case of the representative strain proposed for the identification using the load displacement curve, $F-h$, of a spherical indentation test [8-14], is treated. For this case, many studies were done to propose new representative strains obtained by using finite element simulations and by considering the elastic properties as known. These studies can be divided in two categories. In the first one, the representative strain only depends on the measured parameters $(F$ and $h)[9-12,14]$ and in the second one, it depends on $F, h$ and on the mechanical parameters of the Hollomon hardening law of the material (yield stress $\sigma_{y}$ and work hardening exponent $n$ ) $[8,9,12,13]$. In all these studies, identification methods of these mechanical parameters were proposed.

Using finite element simulations, Taljat et al. [8] proposed two representative plastic strains, maximum and minimum, located near the contact. The choice of Taljat et al. [8] to consider a strain located near the contact was criticized by Lee et al. [9] because of the influence of the friction coefficient between the indenter and the indented material. Lee et al. [9] suggest an optimal data acquisition location, where the strain gradient is the least and the effect of friction is negligible. They proposed a new probing depth that is lower than $20 \%$ of the indenter radius, $R$, from the surface at $0.8 R$, apart from the indentation center. Then, a representative strain determined from finite element simulations was proposed. A maximal ratio of penetration depth to the indenter radius $R, h_{\max } / R=0.12$, was considered sufficient to identify mechanical properties of materials. This choice was later criticized by Lee et al. [13] who demonstrated that for this value of $h_{\max } / R$, multiple plastic properties can give almost the same indentation curve. Therefore, $h_{\max } / R$ was changed to 0.4 by Lee et al. [13]. The authors did not justify their new choice of $h_{\max } / R$. Moreover, they did not justify whether this choice would definitely solve the problem of uniqueness of the solution or not.

Cao and $\mathrm{Lu} \mathrm{[10]} \mathrm{proposed} \mathrm{an} \mathrm{analytic} \mathrm{study} \mathrm{of} \mathrm{the} \mathrm{spherical} \mathrm{indentation} \mathrm{using} \mathrm{dimensional}$ analysis. Using data from Dao et al. [2], the authors proposed a new representative strain definition to obtain a dimensionless function independent from the strain hardening exponent $n$. Two different penetration depths were proposed by the authors to identify two points of the hardening law and thus $\sigma_{y}$ and $n$.

This study was disapproved of by Zhao et al. [11], who considered that it was done on some specific materials and thus, could not be representative of all materials. According to Zhao et al. [11], it is impossible to obtain a dimensionless function independent from the work hardening exponent $n$. Two functions $\Pi$ dependant on the hardening coefficient $n$ were then proposed.

Considering the criticism of Zhao et al. [11], Cao et al. [12] then modified their model [10] for a wider range of materials. They proposed four representative strains for four different penetration depths. More recently, Ogasawara et al. [14] used Cao and Lu's study [10] and proposed a representative strain to simplify the relationship between the energy, the indenter radius and the mechanical parameters.

All the representative strains mentioned above have no physical meaning and were proposed to simplify the identification of mechanical properties using spherical indentation. Moreover, none of the studies concerning the mechanical characterization using the $F-h$ curve [8-14] gave a clear answer on the validity of the identified hardening law and on the sensitivity of the spherical indentation test to the parameters of the hardening law. These parameters were calculated from curve fitting the indentation response of a certain range of material properties. 
The present work is a study of the solution identified for spherical indentation using the $F-h$ curve. A finite element study on elastoplastic materials with the Hollomon hardening law is presented in order to define the precision of the solution that can be determined from the $F-h$ curve. An identification method, based on no assumption, is proposed and an average representative strain is defined. This method leads to the identification of a confidence domain that takes into account the experimental imprecision and the material heterogeneity. The present method is applied for the case of $h_{\max } / R=0.23$ and could be applied for any penetration depth.

\section{2-Material and experimental results}

The material studied was a low-alloy 20MnB5 steel (European Standard EN 10083-3, Steelgrade number: 1.5530 ). The $20 \mathrm{MnB} 5$ is an alloy special steel for quenching and tempering with a chemical composition in weights of $0.191 \% \mathrm{C}, 1.14 \% \mathrm{Mn}, 0.362 \% \mathrm{Si}$, $0.0158 \% \mathrm{P}, 0.0008 \% \mathrm{~S}, 0.25 \% \mathrm{Cr}, 0.0014 \% \mathrm{~B}, 0.039 \% \mathrm{Al}, 0.027 \% \mathrm{Ti}, 0.017 \% \mathrm{Mo}$, $0.025 \% \mathrm{Cu}$ and $0.06 \% \mathrm{Ni}$.

This type of steel was selected to obtain a fine, homogeneous microstructure and thus, a good reproducibility of the indentation tests. Figure 1 represents the microstructure of the 20MnB5 steel and shows a homogeneous distribution of spheroidized carbides in a ferritic matrix.

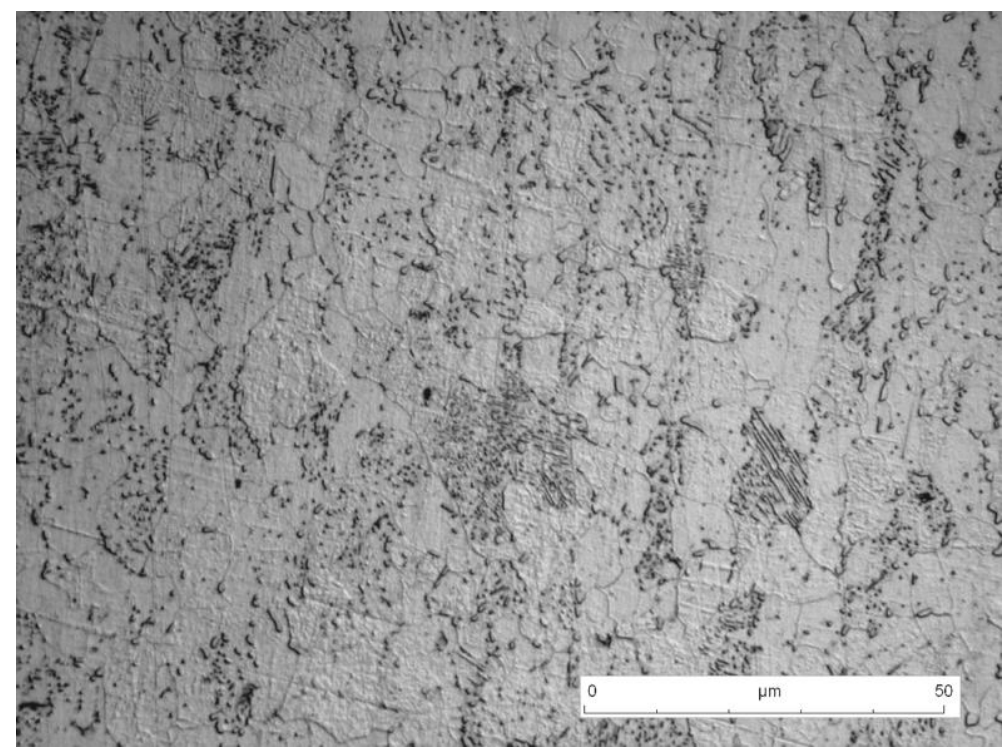

Fig. 1: Microstructure of 20MnB5 steel alloy

The tensile test and indentation specimens were carefully sectioned with a Precision Cut-Off Machine from a hot rolled sheet of $4.5 \mathrm{~mm}$ thickness. The Vickers hardness (10 Kgf) measurements gave $\mathrm{HV}_{10}=155$ for the surface and $\mathrm{HV}_{10}=160$ for the core. The true tensile curves obtained for 20MnB5 steel before necking are represented in Fig. 2. The experimental conditions and measurement method for the tensile test were presented by Moussa et al. [21]. Figure 2 shows that the studied material exhibits a yield stress of about $340 \mathrm{MPa}$ and a nonnegligible work hardening. In general, the work hardening exponent of a material is obtained by using the Hollomon equation. The Hollomon hardening law is often used in evaluating the tensile properties of materials through spherical indentation [3-4, 8-14, 20, 21$]$. This law, obtained from curve fitting the tensile curves, is presented in Fig. 2. This figure shows that the Hollomon equation does not describe the entire flow curve for the 20MnB5 steel. 


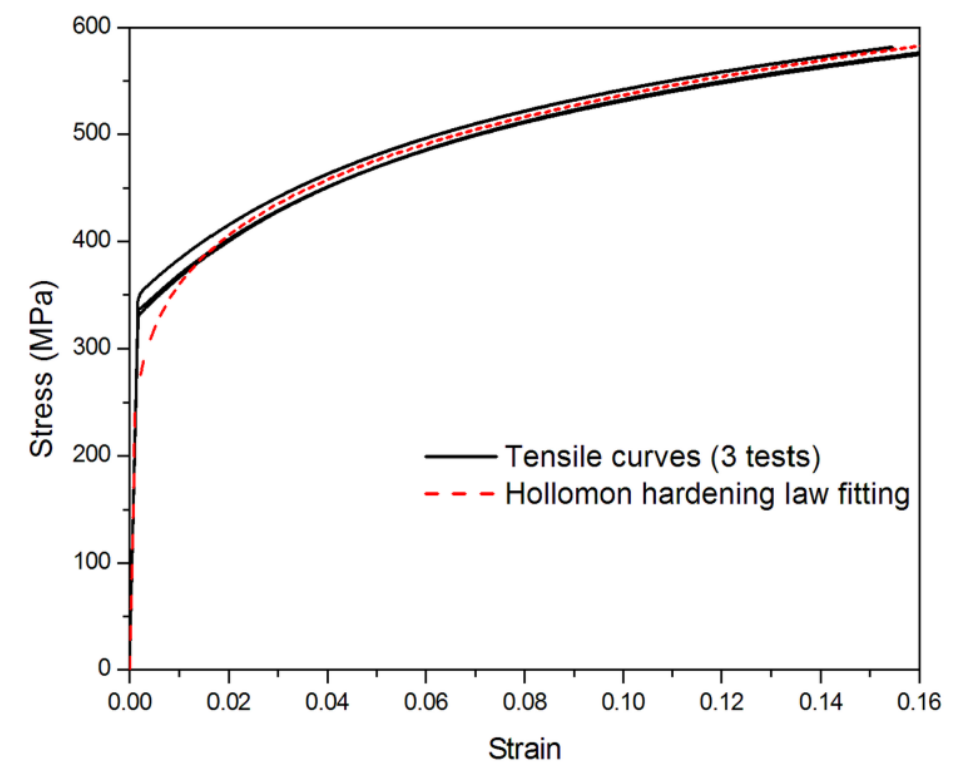

Fig.2: Uniaxial tensile test curves of the 20MnB5 steel

The spherical indentation tests were carried out with a tungsten carbide ball of radius $0.5 \mathrm{~mm}$. The indentation bench used was detailed by Moussa et al. [21]. The core of the material being perfectly homogenous, the indentation tests were done in the core of the material to have a good reproducibility. For the indentation tests in the core, a specimen similar to those used for the tensile test was carefully sectioned with a Precision Cut-Off Machine along the plane perpendicular to the tensile direction. Four spherical indentation curves were obtained from the material. Figure 3 shows that a satisfying reproducibility of the indentation tests was obtained.

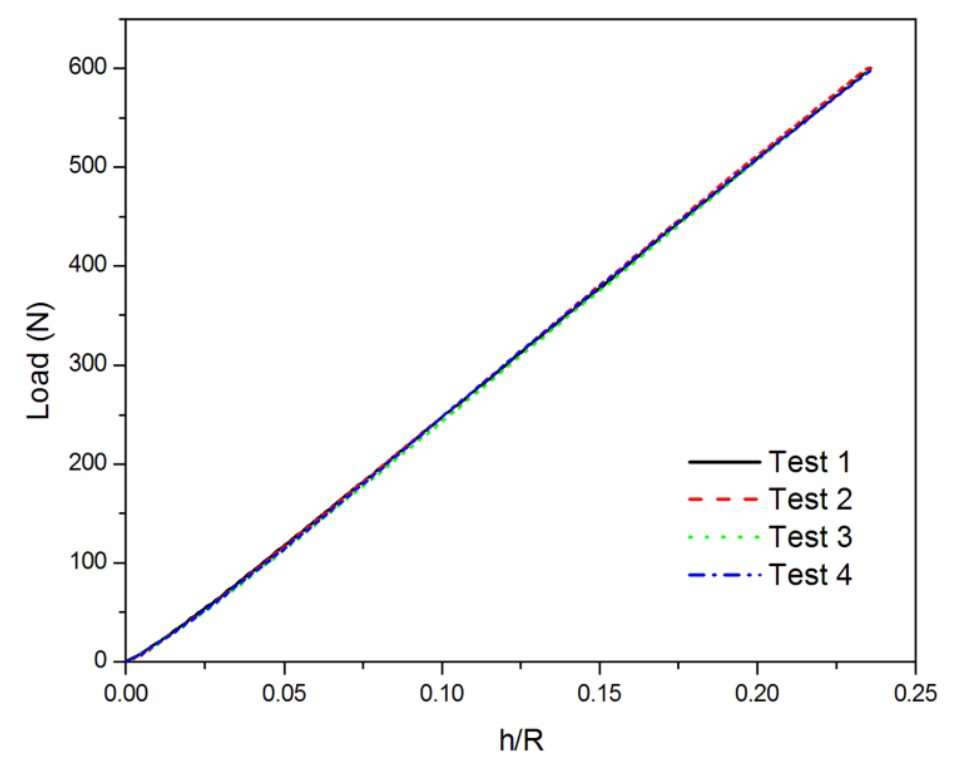

Fig. 3: Spherical indentation curves of the 20MnB5 steel

\section{3-Quantification of the difference between two indentation curves}

In this study, the influence of experimental imprecision and/or material heterogeneity is studied. In order to quantify the difference between two spherical indentation curves with one value, the following root mean square error $\left(E_{R M S}\right)$ was used: 
$E_{R M S}\left(h_{\max } / R\right)=\sqrt{\frac{1}{h_{\max }} \int_{0}^{h_{\max }}\left(F_{1}-F_{2}\right)^{2} d h}$

where $R$ is the radius of the spherical indenter ( $R=0.5 \mathrm{~mm}$ in our case), $h$ is the penetration depth, $h_{\max }$ is the maximal penetration depth, and $F_{1}$ and $F_{2}$ are the load for the two considered curves, respectively.

This error is widely used in inverse analysis [21, 24, 25].

Because of the experimental conditions and the sample's dimensions, $h_{\max } / R=0.23$ was chosen. So, $E_{R M S}$, given in Eq. (1), was calculated between two spherical indentation curves from $h / R=0$ to 0.23 . The proposed method consists of treating the average curve of the four indentation curves (average load calculated for each penetration depth). In order to consider the experimental imprecision and/or material heterogeneity, $E_{R M S}$ was calculated between the average curve, and each of the experimental ones. The values of $E_{R M S}$ are presented in Table 1.

\begin{tabular}{llllll}
\multicolumn{7}{c}{$\boldsymbol{E}_{\boldsymbol{R} M S}(\mathbf{N})$} \\
\hline \hline $\boldsymbol{h}_{\text {max }} / \boldsymbol{R}$ & Test 1 & Test 2 & Test 3 & Test 4 & Maximum \\
\hline \hline 0.23 & 1.17 & 1.97 & 2.50 & 0.85 & 2.50
\end{tabular}

Table 1: Values of root mean square error $\left(E_{R M S}\right)$ between the average curve and the four experimental curves

\section{4-Sensitivity of the hardening law parameters on the load displacement curve}

\section{1-Finite elements model}

An axisymmetric two dimensional finite element model was built up to simulate the indentation test (Fig. 4). The spherical tungsten carbide indenter with $0.5 \mathrm{~mm}$ radius was considered elastic with a Young's modulus of $E=600 \mathrm{GPa}$ and a Poisson's ratio of $v=0.23$. The friction coefficient between the spherical indenter and the sample was fixed at 0.1 .
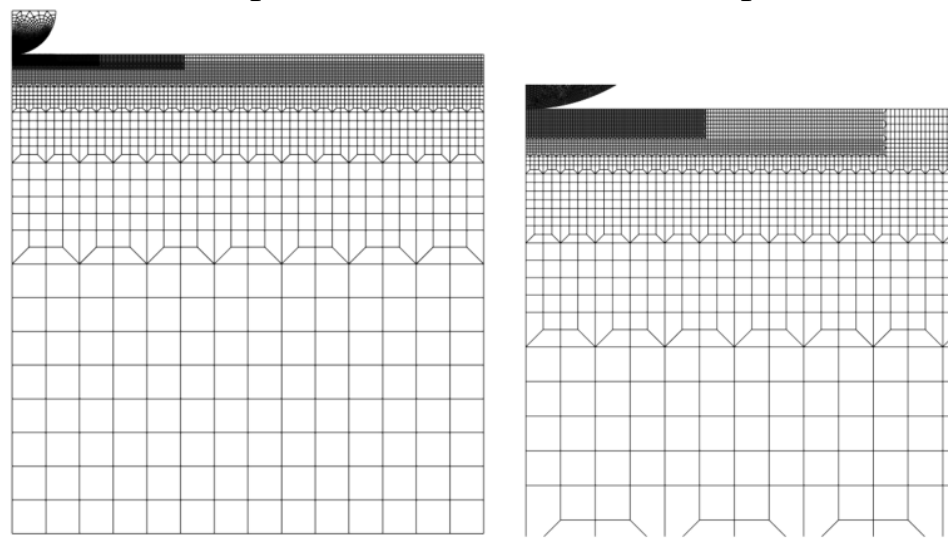

Fig. 4: Detail of the mesh used to simulate the spherical indentation test and a zoom near the contact zone.

Four-noded axisymmetric elements (CAX4) were used with almost 11,000 elements for the sample and 2,000 elements for the indenter. The elements size at the contact zone was about 3 $\mu \mathrm{m}$. The radius of the sample was set large enough so that there would be no effect of outer boundaries. 
The constitutive model of the elastic-plastic-indented material was taken to follow the $\mathbf{J}_{2^{-}}$ associated flow theory with rate independent deformation. The isotropic hardening is described by the following piecewise linear/Hollomon power-law:

$\left\{\begin{array}{cll}\sigma=E \varepsilon & \text { if } & \sigma \leq \sigma_{y} \\ \sigma=E^{n} \sigma_{y}^{(1-n)} \varepsilon^{n} & \text { if } & \sigma \geq \sigma_{y}\end{array}\right.$

where $\sigma_{y}$ is the yield stress of the material and $n$ is the work hardening exponent.

\section{2-Procedure}

The present study consists of calculating the difference between the average experimental curve and various indentation curves obtained from finite element simulations. Therefore, the $E_{R M S}$ given in Eq. (2) was used to study the influence of the hardening law parameters on the indentation curve.

A database was built up from finite element simulations with different combinations of plastic properties, listed in Table 2, and elastic properties corresponding to those of different steels $(E=210 \mathrm{GPa}$ and $v=0.3)$.

\begin{tabular}{cccccc}
\multicolumn{7}{c}{$\sigma_{y}(\mathrm{MPa})$} \\
\hline 190 & 200 & 210 & 220 & 230 & 240 \\
250 & 260 & 270 & 280 & 290 & 300 \\
310 & 320 & 330 & & & \\
\hline \hline 0.09 & 0.095 & 0.1 & 0.105 & 0.11 & 0.115 \\
0.12 & 0.125 & 0.13 & 0.135 & 0.14 & 0.145 \\
0.15 & 0.155 & 0.16 & 0.165 & 0.17 & 0.175 \\
0.18 & 0.185 & 0.19 & 0.195 & 0.2 & 0.205 \\
0.21 & 0.215 & 0.22 & 0.225 & & \\
\hline \hline
\end{tabular}

Table 2: Material Plastic properties used for the finite element simulations of the database.

\section{3-Error distribution}

The $E_{R M S}$ distribution between the average experimental curve and each one of the database curves is presented in Fig. 5. In this figure, the presence of a "valley," in which the values of $E_{R M S}$ are small and almost identical, can be observed. This figure also shows that this "valley" exhibits an obvious direction. A similar correlation between the Hollomon hardening law parameters was observed by Iost co workers [26] for 10,000 perturbed theoretical tensile curves. Despite the small variation in the values of $E_{R M S}$ in the valley observed in Fig. 5, only one minimum $E_{R M S}$ exists for the studied material. The material parameter set $\sigma_{y}=240 \mathrm{MPa}$ and $n=0.182$, that lead to this minimum, are represented by a black dot in Fig. 5. This result proves, once again, the uniqueness of solution when spherical indentation is used for the identification of the yield stress and the work hardening exponent of a material [27].

It can be noticed that the phenomenon observed for spherical indentation is different from the one observed for conical indentation. In the case of conical indentation, Hollomon hardening laws that exhibit the same stress for a specific value of strain (called representative strain) give the same indentation curve $[2,28]$. Therefore, no unique hardening law can be identified from a conical indentation curve, which is not the case of the spherical indentation. 


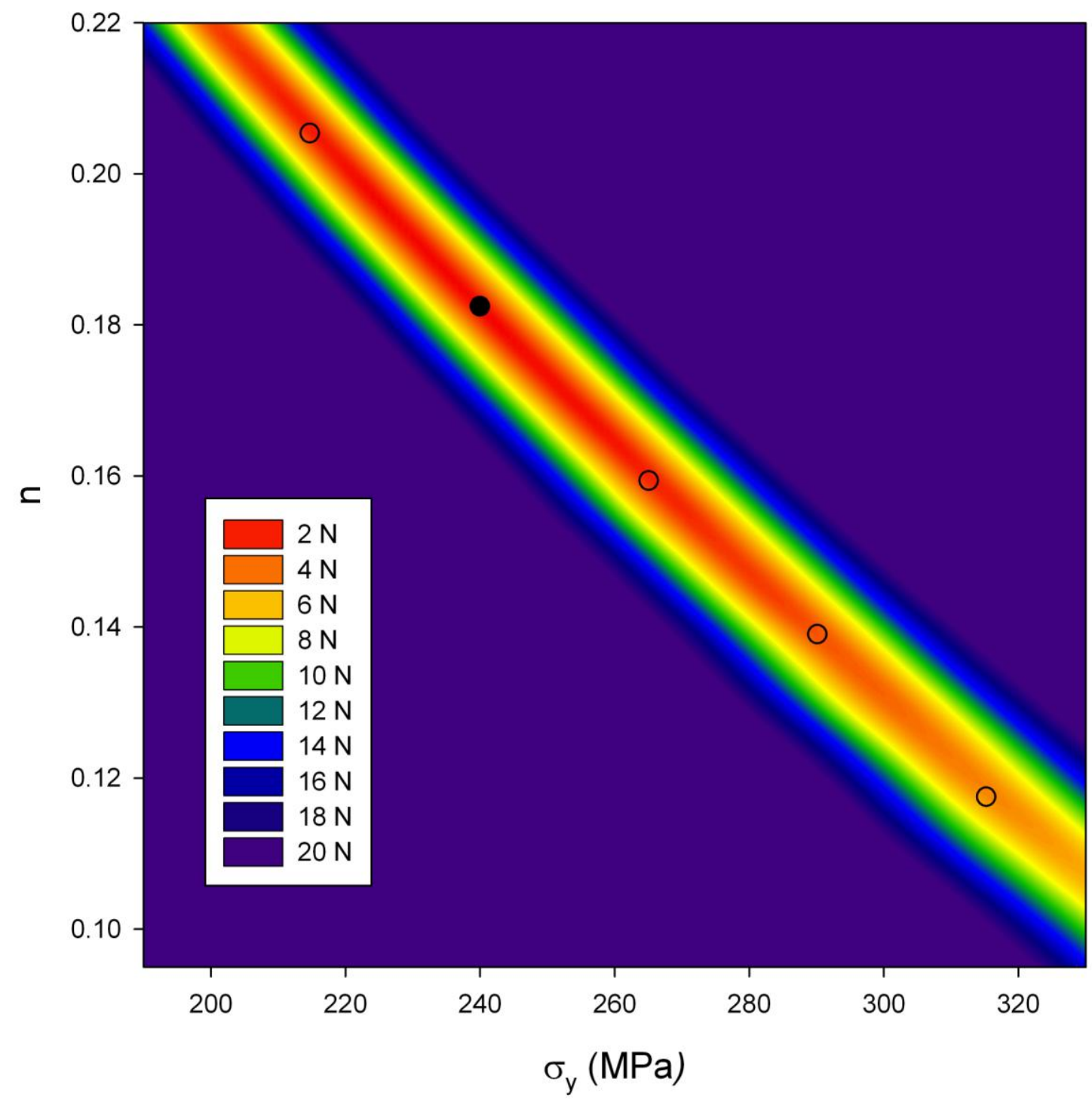

Fig. 5: Root mean square error $\left(E_{R M S}\right)$ distribution between the average experimental indentation curve and each one of the indentation curves of the database. The small circles represent the couples of parameters that are located at the bottom of the "valley".

\section{5-Identification method}

\section{1-Reference change}

The bottom of the valley, in Fig. 5, is incurved in the $\sigma_{y}-n$ diagram. In order to simplify the form of the valley, a reference change is proceeded from $\sigma_{y}-n$ diagram to $k-n$ diagram where

$k=(1-n) \ln \frac{\sigma_{y}}{E}$

The $E_{R M S}$ distribution in $k-n$ diagram is presented in Fig. 6 . We observe in this figure that the valley is no longer incurved. The bottom of the valley in the $k-n$ diagram follows a straight line. From Fig. 6, one can also notice that the $E_{R M S}$ distribution in the $k-n$ diagram takes a particular form: a cone with an elliptical base. This result justifies the proposed change of 
variable made in order to simplify the form of this valley. Further explanations about the reason why the bottom of the valley follows a straight line in the $k-n$ diagram will be given in section 6 .

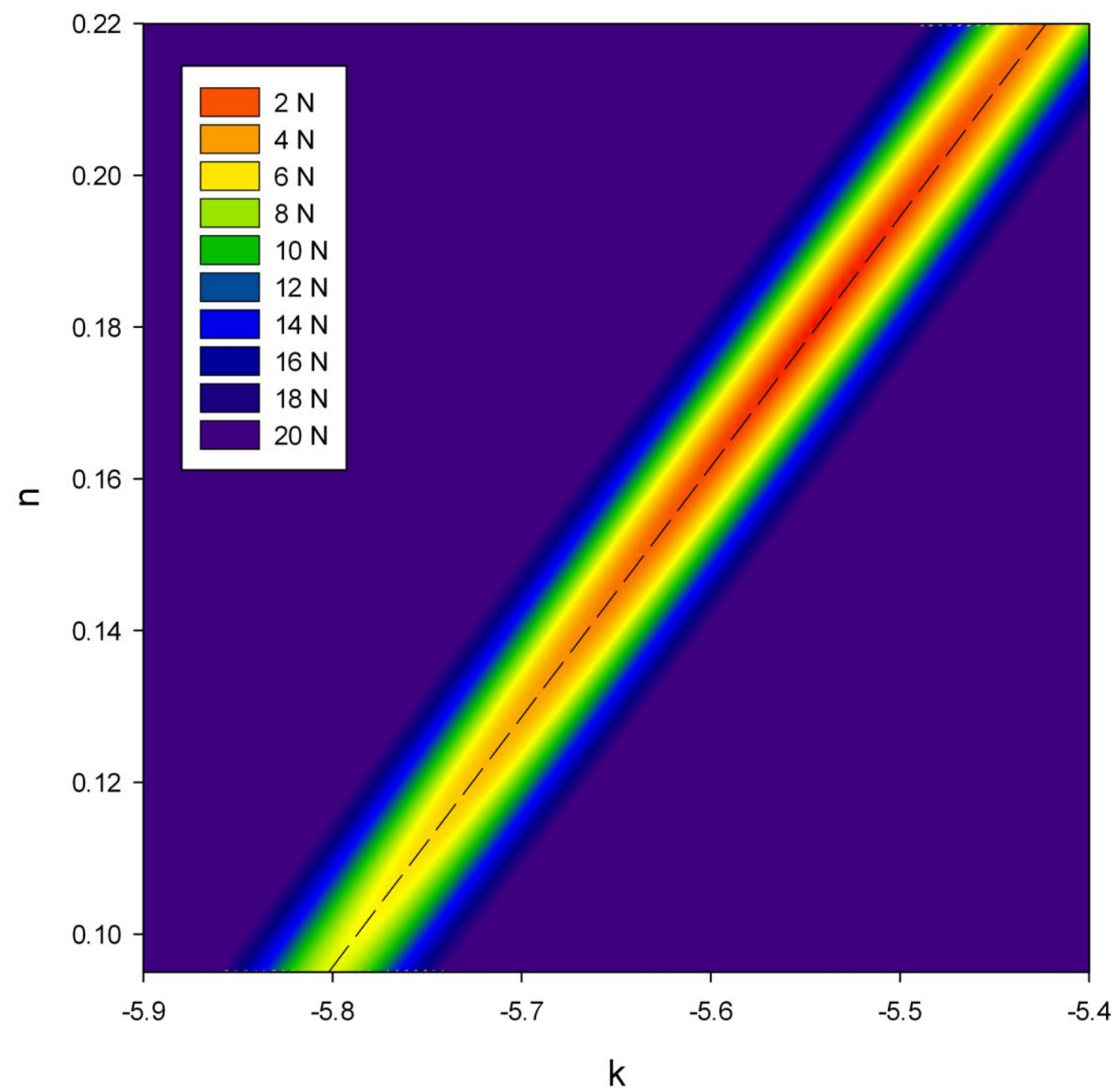

Fig. 6: Root mean square error $\left(E_{R M S}\right)$ distribution between the average experimental indentation curve and each one of the indentation curves of the database in the $k-n$ diagram.

\section{2-Equation for $E_{R M S}$ distribution}

The aim of the study presented in this paragraph is to define an analytic function that can describe the $E_{R M S}$ distribution with no need to accomplish a large number of finite element simulations to form a database. The cone with the elliptical base can be defined with five parameters as shown in Fig. 7:

$-k_{0}$ and $n_{0}$ : these parameters correspond to the location of the summit of the cone. They give the minimal value of $E_{R M S}$, corresponding to the solution (Fig. 7).

$-X$ and $Y$ : these parameters correspond to the major and the minor semi-axes of the cone for each value of $E_{R M S}$ as follows: $E_{R M S} . X$ and $E_{R M S} . Y$ (Fig. 7).

$-\theta_{a R}$ : angle between the major axis of the ellipse and the $k$ axis (Fig. 7). 

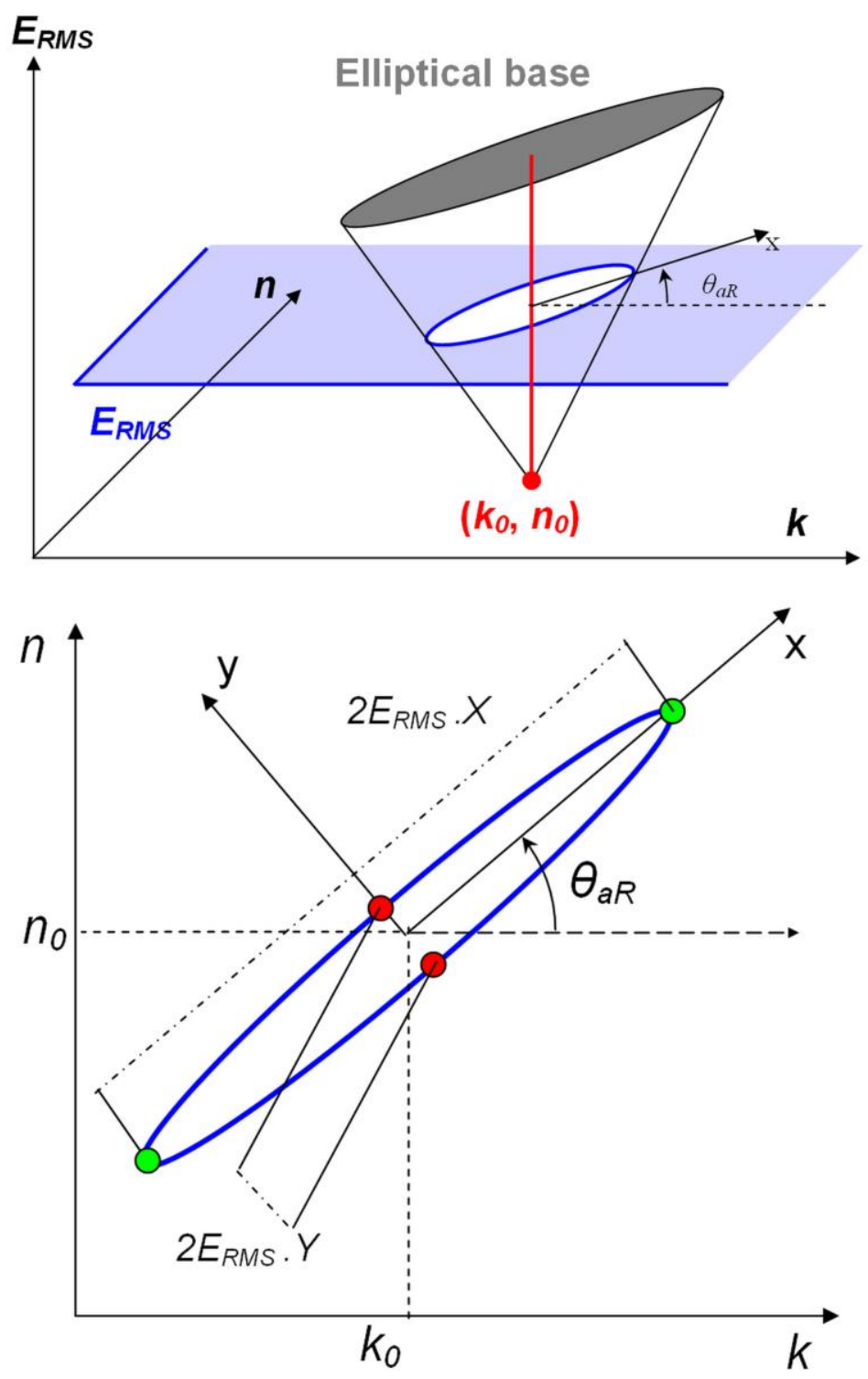

Fig.7: (a) Schematic of the cone with the elliptical base that describes the $E_{R M S}$ distribution in $k$ - $n$ diagram.

(b) Schematic of the intersection between the cone with the elliptical base and a plane defined with a value of $E_{R M S}$.

Using these five parameters, the $E_{R M S}$ distribution in the $k-n$ diagram can be written as follows:

$$
E_{\text {ellipse }}=\sqrt{\left(\frac{x}{X}\right)^{2}+\left(\frac{y}{Y}\right)^{2}}
$$

with

$x=\left(k-k_{0}\right) \cos \theta_{a R}+\left(n-n_{0}\right) \sin \theta_{a R}$

and

$y=-\left(k-k_{0}\right) \sin \theta_{a R}+\left(n-n_{0}\right) \cos \theta_{a R}$

The dimensional unit of $E_{\text {ellipse }}$ is the same as $E_{R M S}$ (Newton, in our case). The dimensional units of $X$ and $Y$ are the inverse of the dimensional unit of $E_{\text {ellipse. }}$. 
From these equations, it can be written as:

$$
E_{\text {ellipse }}=f\left(n_{0}, \sigma_{y 0}, \theta_{a R}, X, Y\right)
$$

With $\sigma_{y 0}=E \exp \left(k_{0} /\left(1-n_{0}\right)\right)$

Note: The representation of the surface defined in Eq. (4) in the $\sigma_{y}-n$ diagram is a cone with an incurved elliptical base. In the following parts of this article, the $E_{R M S}$ distribution will be represented in the $\sigma_{y}$ - $n$ diagram to simplify the observations.

\section{3-Identification}

The five parameters, $X, Y, n_{0}, \sigma_{y 0}$ and $\theta_{a R}$, are obtained from the minimal value of the following cost function:

$E=\sum\left(E_{R M S}^{i}-E_{\text {ellipse }}^{i}\right)^{2}$ with $E_{R M S}^{i}-E_{\text {ellipse }}^{i}=0$ if $\quad E_{R M S}^{i} \geq E_{\text {critical }}$

where $i$ corresponds to each case of the material parameter sets $\left(\sigma_{y}, n\right)$ that were chosen for the database. It should be noticed that the elliptical cone form was assumed to be the form of the $E_{R M S}$ distribution near the solution, i.e where the valley exists. For this reason, $E_{\text {critical }}$ was defined to use only the material parameter sets that are near the solution to correctly determine the five parameters of the elliptical cone. In this study, $E_{\text {critical }}=30 \mathrm{~N}$ was chosen.

The comparison between $E_{R M S}$, obtained from the database, and $E_{\text {ellipse }}$ obtained from Eq. (4) is presented in Fig. 8. In Fig. 8, it is clearly shown that the $E_{\text {ellipse }}$ distribution given by Eq. (4) superimposes perfectly onto the $E_{R M S}$ distribution. The result given in this figure proves that the considered assumption on the form of the valley is correct and that the valley takes the form of a cone with an elliptical base.

Note: The small differences that can be observed in Fig. 8 are mainly due to the lack of FE simulations in the database. 


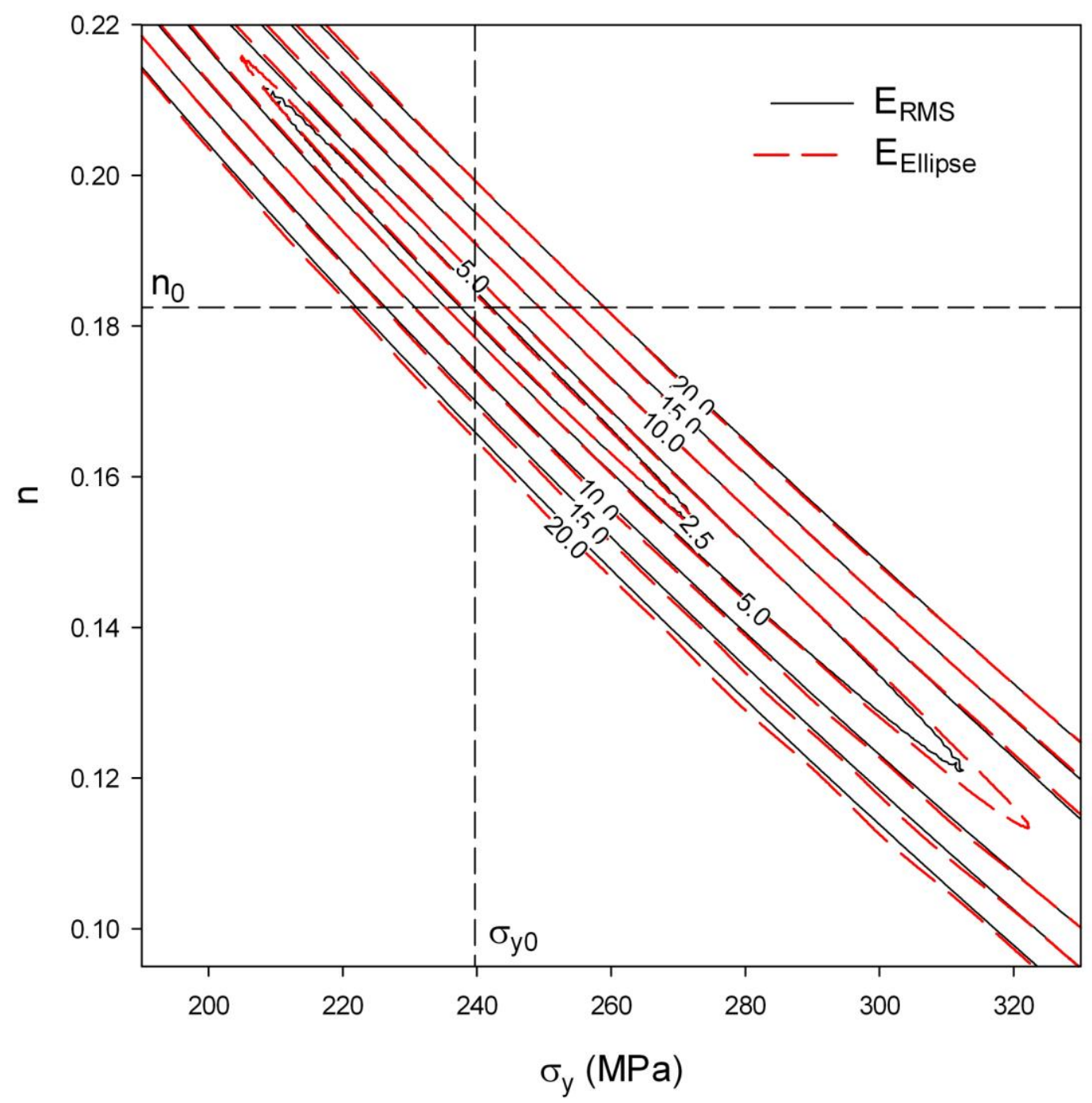

Fig. 8: Comparison between the $E_{R M S}$ distribution (from the database) and $E_{\text {ellipse }}$ (from Eq. (4))

From Eq. (4), $\sigma_{y 0}=240 \mathrm{MPa}$ and $n_{0}=0.182$ were determined using the average experimental $F-$ $h$ curve. The hardening law that corresponds to these parameters is presented in Fig. 9 (red dots) in comparison with the tensile test curves. In this figure, it can be seen that the identified hardening law with spherical indentation is very close to the tensile test curves, which proves once again that the spherical indentation technique can be used to identify the hardening laws of materials. 


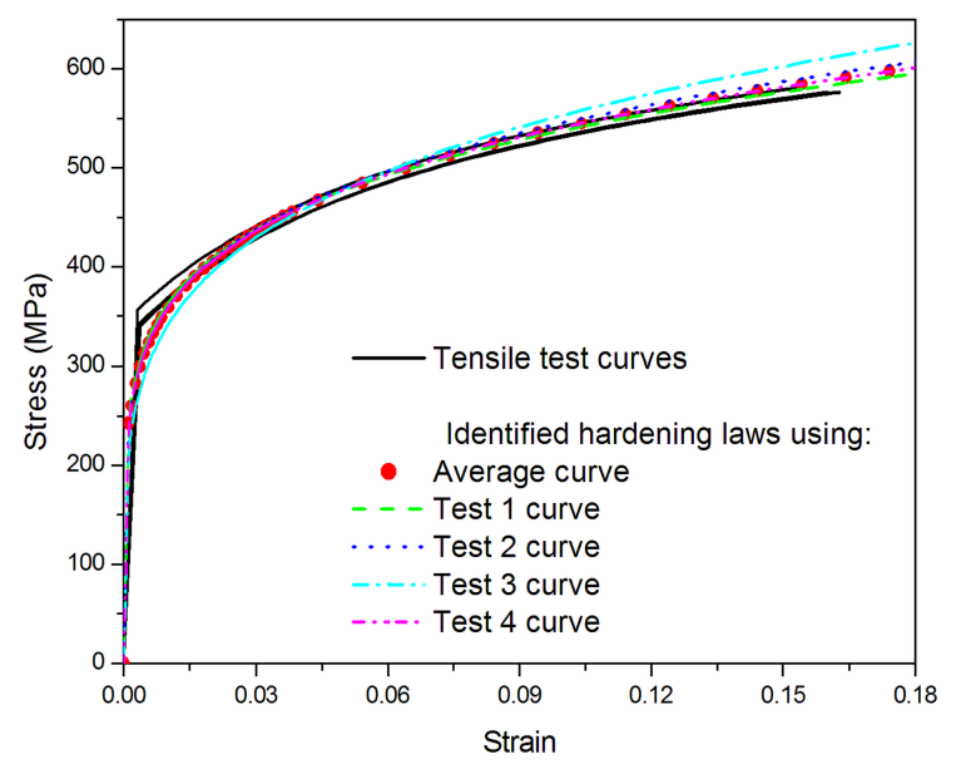

Fig. 9: Comparison between the tensile test curves and the identified hardening laws with the elliptical cone definition from the experimental average indentation curve and the four experimental indentation curves

\subsection{Discussion about the identification method}

A large number of the proposed identification methods are based on the determination of a representative strain. According Lee et al. [9, 13], the representative strain was determined to be the strain of a specific zone of the indented material. No physical justification was given to explain the reason why this strain can be considered as representative of spherical indentation. As for the other methods $[4,10-12,14]$, the representative strain was defined to simplify the mathematical formulation that links the $F-h$ curve with the material parameters. In all these studies, the use of the representative strain serves as a mathematical trick having no physical basis. Unlike these methods, the identification method proposed in this section is not based on the determination of a subjective representative strain. Using Eq. (4), the distribution of the error between an experimental curve and FE simulation curves can be determined. From this equation, the set of parameters that gives the minimum value of error, which corresponds to the solution, can be identified.

Therefore, the proposed method leads to the identification to the Hollomon hardening law corresponding to the material parameter set $\left(\sigma_{y o}, n_{o}\right)$. However, no indication about the most precise part of the identified hardening law is given with the proposed method at this stage of the study. Moreover, no information on the sensitivity of the acquired plastic properties $\left(\sigma_{y}\right.$ and $n$ ), associated with material heterogeneity and experimental imprecision is given.

In order to study this sensitivity, the proposed method was applied on the four experimental indentation curves (Fig. 3). The identified parameters are presented in Table 3 and the corresponding hardening laws in Fig. 9. Which one is to be considered as solution and how to proceed to determine a domain that includes all the solutions? Should we consider that the domain defined with the square determined from the minimal and the maximal values of $\boldsymbol{\sigma}_{\boldsymbol{y}}$ and $\mathrm{n}\left(210<\sigma_{y}<252\right.$ and $\left.0.171<\mathrm{n}<0.21\right)$ includes all the solutions?

The part of the hardening law that is identified with the highest precision and answers to these questions are given in the following sections.

$h_{\max } / R=0.23$

\begin{tabular}{llll|l} 
Test 1 & Test 2 & Test 3 & Test 4 & Average $F$ - $h$
\end{tabular}




\begin{tabular}{ccccc||c}
$\sigma_{\boldsymbol{y}}(\mathbf{M P a})$ & 251.94 & 242.08 & 210.21 & 244.16 & 239.83 \\
$\boldsymbol{n}$ & 0.1716 & 0.1821 & 0.2103 & 0.1787 & 0.182
\end{tabular}

Table 3: $\sigma_{y}$ and $n$ identified from the average and from the four experimental indentation curves.

\section{6- Average representative strain}

As shown in Figs. 5 and 6, the distribution of the error takes the form of a valley in the $[k, n]$ or $\left[\sigma_{Y}, n\right]$ diagram. The material parameter sets $\left(\sigma_{y}, n\right)$ located in the bottom of this valley give hardening laws that lead to $F-h$ curves which are very close to the $F-h$ experimental curve. Some of those hardening laws (small circles in Fig. 5) are presented in Fig. 10. From this figure, it can be noticed that these hardening intersect in a same point. We define the "average representative strain" $\varepsilon_{a R}$ as the abscissa of the intersection point. In the studied case, its value is 0.0487 . The stress equivalent to the average representative strain is called $\sigma_{a R}$. In the bottom of the valley, all the Hollomon hardening laws that intersect the stress-strain curve corresponding to the solution at $\left[\varepsilon_{a R}, \sigma_{a R}\right]$ lead to indentation curves close to the indentation curve corresponding to the solution. Hence, the $F-h$ curve is mostly influenced by the part of the hardening law which is located around the average representative strain, $\varepsilon_{a R}$. When a material is characterized using the $F-h$ curve, it is this part of the Hollomon hardening law that is mostly characterized and the better identified.

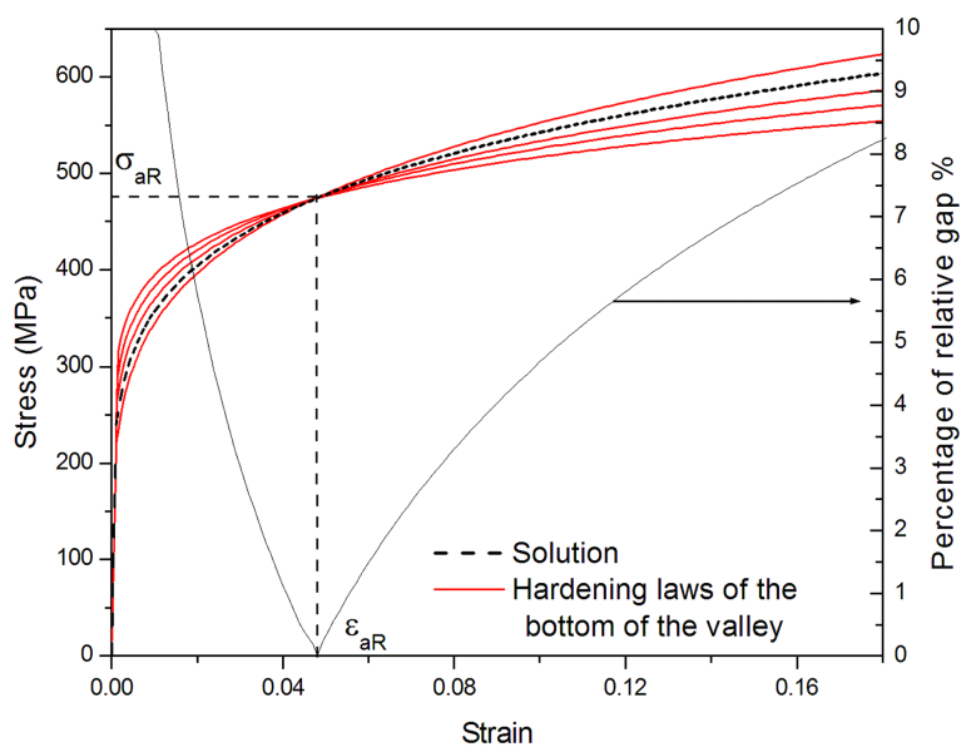

Fig. 10: Hardening laws of the materials for which the material parameter sets $\left(\sigma_{y}, n\right)$ are located in the bottom of the valley (represented by small circles in Fig. 5) and the percentage of the maximal relative gap between these curves.

When $\varepsilon=\varepsilon_{a R}$, Eq. (2) becomes for the plastic part of the stress-strain curve:

$\sigma=\sigma_{y}^{(1-n)} E^{n} \varepsilon_{a R}^{n}$

From Eq. (3), we can easily obtain

$\ln \frac{\sigma_{a R}}{E}=(1-n) \ln \frac{\sigma_{y}}{E}+n \ln \varepsilon_{a R}$

If we use the reference change proceeded in section 5.1, i.e $k=(1-n) \ln \frac{\sigma_{y}}{E}$, we obtain 
$n=-\frac{1}{\ln \varepsilon_{a R}} k+\frac{\ln \frac{\sigma_{a R}}{E}}{\ln \varepsilon_{a R}}$

For the material parameters sets $\left(\sigma_{y}, n\right)$ that are located in the bottom of the valley, $\varepsilon_{a R}$ and $\sigma_{a R}$ are constants and Eq. (11) becomes a linear equation in $k$ - $n$ diagram with a slope of $-\frac{1}{\ln \varepsilon_{a R}}$

for materials with the same $E$. This result explains why the bottom of the valley follows a straight line in the $k$ - $n$ diagram as it was shown in Fig. 6.

The direction of the bottom of the valley defined with the angle $\theta_{a R}$ corresponds to the slope of Eq. (11). Hence, we can write

$\varepsilon_{a R}=\exp \left(-\frac{1}{\tan \theta_{a R}}\right)=\exp \left(-\frac{\partial k}{\partial n}\right)$

$\theta_{a R}$ is calculated using the elliptical cone definition (Eqs. (4, 5, 6). From Eq. (12), the determined average representative strain is $\varepsilon_{a R}=0.0487$. As expected, this value corresponds to the strain for which all the hardening laws of the bottom of the valley intersect (see Fig. $10)$.

We would like to state that $\varepsilon_{a R}$, defined in this study, is dependent on the measured parameters during indentation ( $F$ and $h$ in our case) and on the definition of the error (root mean square in our case). Moreover, $\varepsilon_{a R}$ is characteristic of the whole indentation response from $h=0$ to $h=h_{\max }$. For this reason, $\varepsilon_{a R}$ is called "average representative strain" by the authors of this study.

As already mentioned, the representative strains proposed earlier [1-19] are all dependent on the choice of the functional parameters that were used to describe the indentation process and are not strictly determined from the material response. The proposed average representative strain is strictly determined from an observation of the material response to the spherical indentation. Even more, in the studies concerning spherical indentation [1, 8-13, 15-17, 19], the representative strain is obtained for one specific state of deformation, i.e., for $h / R$ or $a / R$ fixed. For example, Tabor proposed the following representative strain $\varepsilon_{R}=0,2 a / R$. The use of this equation leads to a representative strain characteristic of the response of the material at one specific state of deformation, which means that the history of deformation is not considered. The proposed average representative strain is characteristic of the whole indentation response and not only of one specific state of deformation. Lastly, this average representative strain is not used to identify the Hollomon hardening law of the material but to obtain more information of the most precise part of the identified hardening law.

In the following section, the proposed identification method and the average representative strain are used to analyse the sensitivity of the acquired plastic properties $\left(\sigma_{y}\right.$ and $\left.n\right)$, associated with material heterogeneity and experimental imprecision.

\section{7- Experimental imprecision and material heterogeneity}

Experimental measurement imprecision and material heterogeneity make it impossible to obtain two experimental indentation curves which are perfectly similar. In a review of several proposed methods for estimating hardening laws from spherical indentation $F$ - $h$ curves, published in 2004 [26], Cheng and Cheng mentioned: "However, since measurement errors in $F i$ and hi can cause errors in the estimation of $E, \sigma_{y}, v$ and $n$, it is insufficient to report the best estimates of $E, \sigma_{y}, v$ and $n$ without reporting their errors. Detailed sensitivity analyses of the effect of various errors would thus be necessary. Such robust techniques for ascertaining stress-strain relationship as well as their confidence levels will likely be available in the future." The question is how to proceed to consider multiple experimental indentation curves. Is it 
necessary to use all the curves in order to accomplish a statistical study so the result can describe the real behavior of the material? In this section, the differences between the four $F-h$ curves and their influence on the identified hardening law are studied. A solution and confidence domains are proposed to identify the plastic properties of a material while taking into account the influence of the experimental imprecision and material heterogeneity. This way the identified solution will not be a unique solution, which corresponds to a single hardening law, but a domain that gathers all possible solutions. To do so, the maximal value of the errors between the average $F-h$ curve and each one of the four experimental $F-h$ curves is used (Table 1). This maximal error characterizes the influences of the experimental imprecision and the material heterogeneity on the $F-h$ curves. Hence, in the following section, the average curve and the maximal experimental error are used to identify the plastic properties of a material taking into account the experimental imprecision and the material heterogeneity.

\subsection{Solution domain}

The isovalue of $E_{\text {ellipse }}=2.5 \mathrm{~N}$ (maximal experimental error, see Table 1) is presented in Fig. 11. All the material parameter sets $\left(\sigma_{y}, n\right)$ located inside this isovalue curve give hardening laws that lead to $F-h$ curves with a $E_{R M S}$ value lower than $2.5 \mathrm{~N}$. Because this value is the maximal error between the average curve and the experimental ones, this isovalue curve includes all the material parameter sets $\left(\sigma_{y}, n\right)$ that can be considered as solutions. Therefore, this isovalue curve is defined by the authors as the solution domain. In order to validate the use of the solution domain, the four material parameter sets $\left(\sigma_{y}, n\right)$ identified from each one of the experimental curves (Table 3), are presented with colored dots in Fig. 11. As seen from Fig. 11, they are located inside the solution domain, which means that the solution domain includes all possible solutions. 


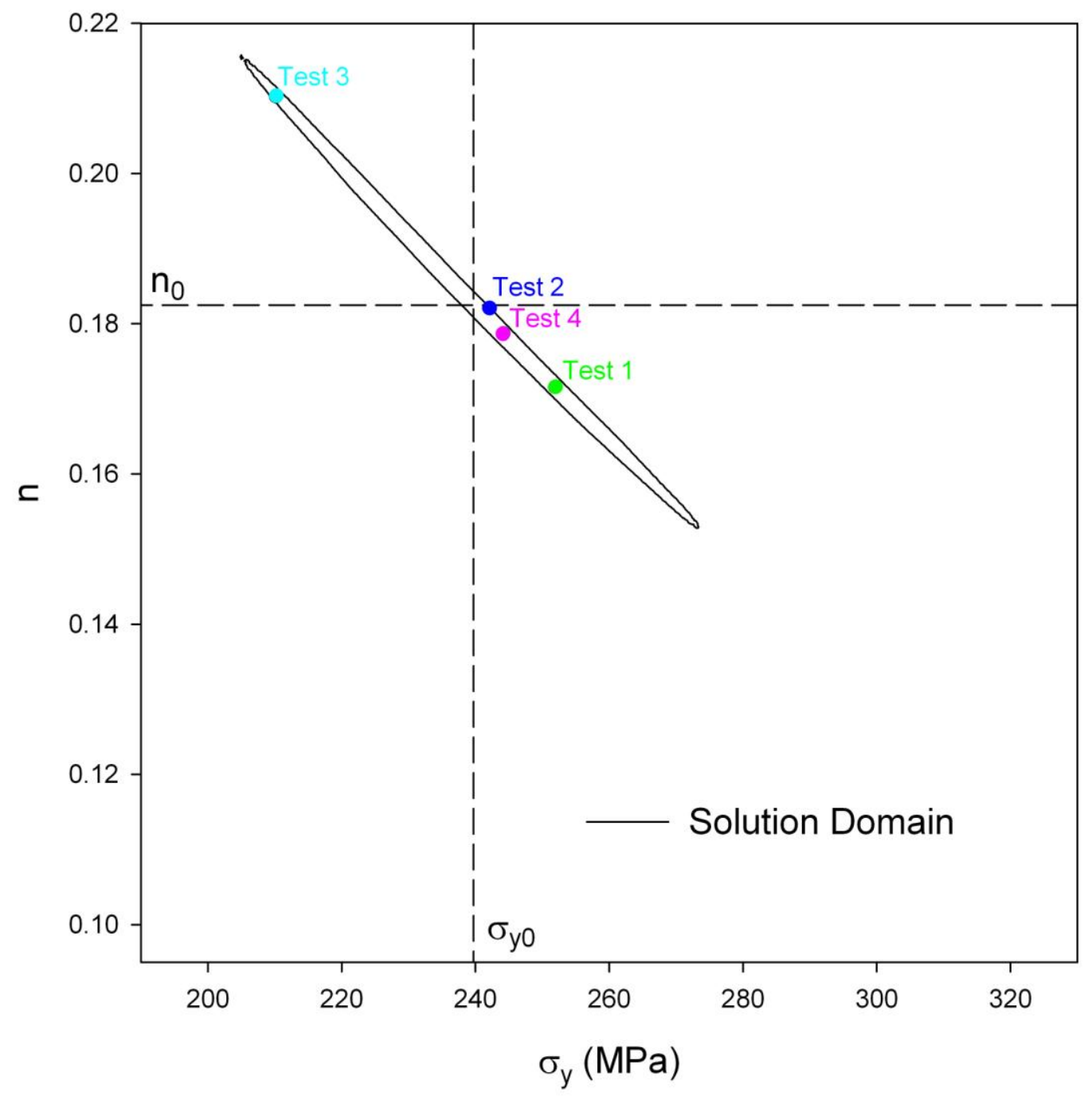

Fig. 11: Solution domain with the material parameter sets $\left(\sigma_{y}, n\right)$ identified from the average and the four experimental indentation curves.

Figure 3 shows that a satisfactory reproducibility of the four $F-h$ curves is obtained. On the other hand, Table 3 shows that the difference between the material parameters identified from these curves is not negligible. These results demonstrate the high sensitivity of the instrumented indentation test to the material parameter sets $\left(\sigma_{y}, n\right)$. The comparison between the tensile test curves and the identified hardening laws obtained from these material parameter sets shows, however, that they represent, in a satisfying way, the hardening law of the material obtained from the tensile test curve (Fig. 9). A high degree of correlation between $\sigma_{y}$ and $n$ (correlation coefficient close to -1 ) is observed in Fig. 11. This result shows that the solution domain is smaller than the domain defined with the square that can be determined from the minimal and the maximal values of $\sigma_{y}$ and n, i.e $210<\sigma_{y}<252$ and $0.171<n<0.21$ (see Table 3).

\subsection{Confidence domain}

In this paragraph, a procedure is developed to represent the domain equivalent to the solution domain in the stress-strain diagram. The solution domain is represented in Fig. 12 in $k-n$ and $\sigma_{y}-n$ diagrams. This domain includes multiple parameters of hardening laws. The envelopes 
of these hardening laws are represented in Fig. 13. The domain delimited by the envelopes is defined by the authors as the confidence domain. In Fig. 12, the thickness of this domain is presented. This thickness is minimal when $\varepsilon=\varepsilon_{a R}$, which shows that the most precise part of the identified hardening law is obtained around the average representative strain while taking into account the influence of the experimental imprecision and material heterogeneity.

The red and green dots represented in Fig. 12 (points a, b, c and d) are the intersection points between the ellipse (in $k-n$ diagram) and its two principle axes. The hardening laws corresponding to these dots are represented in Fig. 13b. As can be seen, the domain limited by the envelopes of these hardening laws is almost the same as the confidence domain (difference between the stresses smaller than $0.49 \%$ for the studied case). Therefore, we assume that the confidence domain is determined from the hardening laws corresponding to points $\mathrm{a}, \mathrm{b}, \mathrm{c}$ and $\mathrm{d}$ represented in Fig. 12. The hardening laws corresponding to points $\mathrm{a}$ and $\mathrm{b}$ intersect at strain, and those corresponding to points $b$ and $c$ intersect at strain $\varepsilon_{b c}$ (Fig. 13b). In the same way, the hardening laws corresponding to points $\mathrm{c}$ and $\mathrm{d}$ intersect at strain $\varepsilon_{c d}$ and those corresponding to points $\mathrm{d}$ and a intersect at strain $\varepsilon_{d a}$. Fig. 13 shows $\varepsilon_{a b}=\varepsilon_{c d}$ and $\varepsilon_{b c}=$ $\varepsilon_{d a}$.
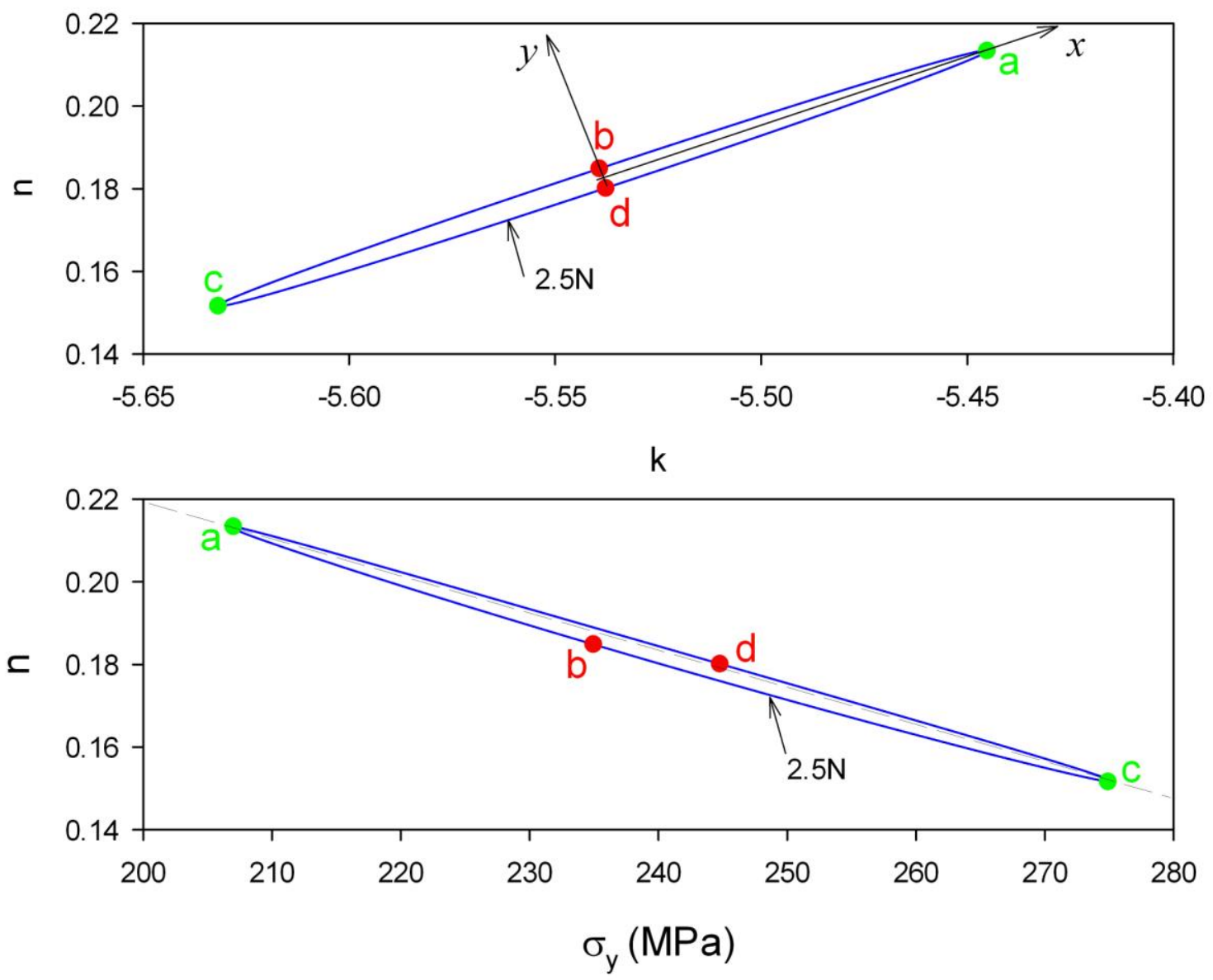

Fig. 12: Solution domain with the intersection points between its perimeter and its principle axes, in $\sigma_{y}-n$ and k-n diagram. 


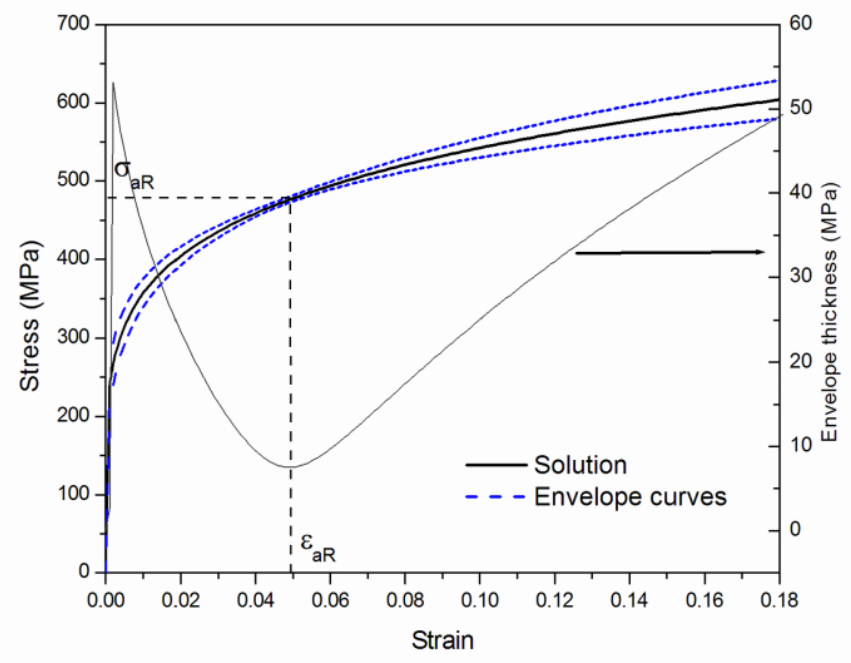

(a)

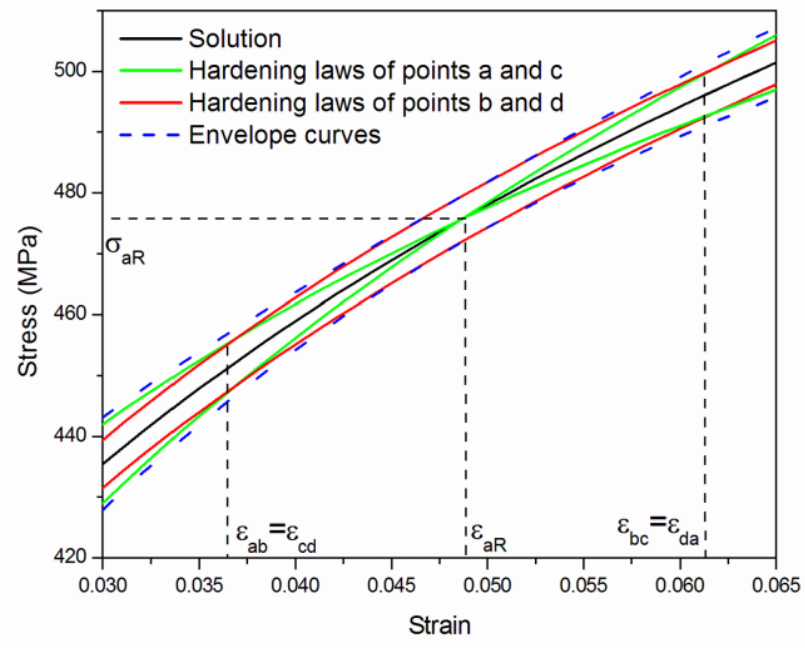

(b)

Fig. 13: (a) Identified solution and confidence domain limited with the envelope

(b) Zoom around $\varepsilon_{a r}$ : Identified solution, confidence domain limited with the envelope and hardening laws corresponding to points $a, b, c$ and $d$ represented in Fig. 12

Since the confidence domain is defined with the hardening laws corresponding to points $a, b$, $\mathrm{c}$ and $\mathrm{d}$, the solution domain is reduced from an ellipse to a rhombus as presented in Fig. 14. 


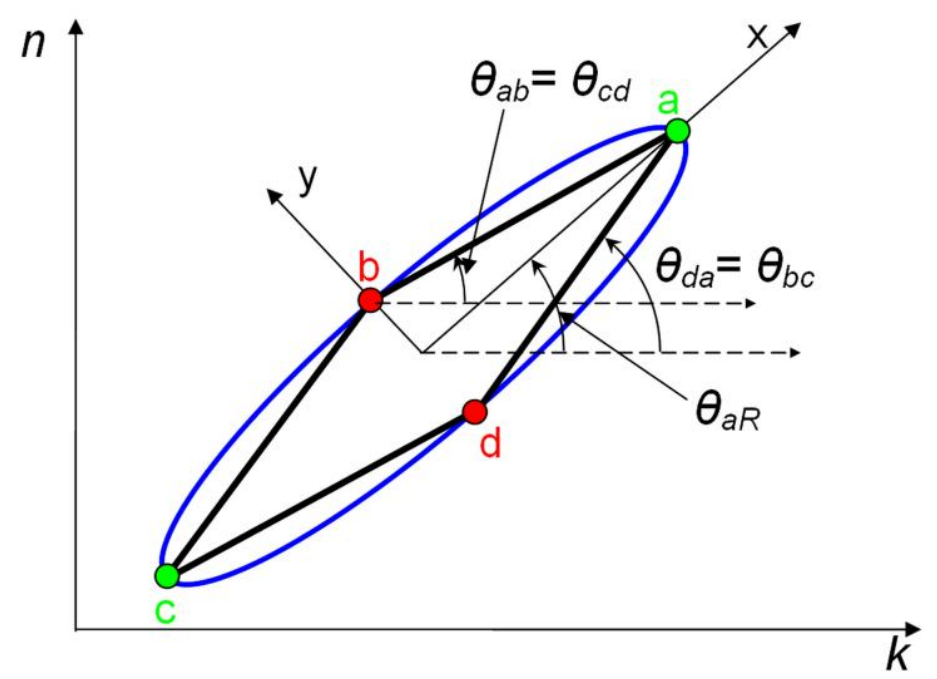

Fig. 14: Simplification of the solution domain from an ellipse to a rhombus.

This new form of the solution domain makes the determination of the confidence domain easier.

As already presented, the direction of the principle axis of the rhombus is connected to the average representative strain. Using the same approach for $\varepsilon_{a b}$ the direction of the line segment $[a b]$ leads to the strain for which the hardening laws, corresponding to points $a$ and $b$ intersect. In the same way as for $\varepsilon_{b c}$, the direction of the line segment [bc] leads to the strain for which the hardening laws, corresponding to points $b$ and $c$ intersect. Since for a rhombus, the opposite sides are parallel, the directions of the four sides are defined by two angles as shown in Fig. 14. Thus, according to Eq. (12) and Fig. 14, $\varepsilon_{a b}$ and $\varepsilon_{b c}$ can be calculated using the following equations:

$$
\begin{gathered}
\varepsilon_{a b}=\varepsilon_{c d}=e^{-\frac{1}{\tan \theta_{a b}}} \\
\varepsilon_{b c}=\varepsilon_{d a}=e^{-\frac{1}{\tan \theta_{b c}}}
\end{gathered}
$$

Because of the geometry of the rhombus and using Taylor series, it can be easily demonstrated that

$$
\begin{aligned}
& \varepsilon_{a b}=\varepsilon_{c d} \approx \varepsilon_{a R}-\Delta \varepsilon_{a R} \\
& \varepsilon_{b c}=\varepsilon_{d a} \approx \varepsilon_{a R}+\Delta \varepsilon_{a R}
\end{aligned}
$$

with

$$
\Delta \varepsilon_{a R}=\varepsilon_{a R}\left(1+\left(\ln \varepsilon_{a R}\right)^{2}\right) \arctan \frac{Y}{X}
$$

Equation (15) shows that $\varepsilon_{a b}$ and $\varepsilon_{b c}$ are symmetrically located with respect to $\varepsilon_{a R}$. The confidence domain is defined, between $\varepsilon_{a b}$ and $\varepsilon_{b c}$, by the hardening laws corresponding to points $b$ and $d$ represented in Figs. 12 and 14. The parameters of the hardening laws of points $\mathrm{b}$ and $\mathrm{d}$ are:

$$
\text { point b: } \begin{aligned}
& k=k_{0}-E_{R M S} Y \sin \theta_{a R} \\
& n=n_{0}+E_{R M S} Y \cos \theta_{a R}
\end{aligned} \quad \text { point d: } \begin{aligned}
& k=k_{0}+E_{R M S} Y \sin \theta_{a R} \\
& n=n_{0}-E_{R M S} Y \cos \theta_{a R}
\end{aligned}
$$

Any Hollomon hardening law located inside the confidence domain between $\varepsilon_{a b}$ and $\varepsilon_{b c}$, is located inside the confidence domain for every value of strain. Hence, the confidence domain is defined with the hardening laws corresponding to points $b$ and d, given in Eq. (17), between $\varepsilon_{a b}$ and $\varepsilon_{b c}$. 
In section 5.2, Eq. (7) was used to identified the parameter of the hardening law of the material $n_{0}, \sigma_{y 0} . \theta_{a R}, X, Y$ parameters were also identified but not used. In this section, using the five identified parameters in Eqs. (12, 15-17), the confidence domain is determined, taking into account the experimental imprecision and/or the heterogeneity of the material.

The curves obtained with the tensile test, the confidence domain and the five hardening laws identified (using the average $F-h$ curve and the four $F-h$ curves) are represented in Fig. 15. The hardening laws identified using the four $F-h$ curves are all located inside the confidence domain which means that using the average curve, the proposed procedure, using Eq. $(7,12$, 15-17), enables the material to be identified, taking into account the experimental imprecision and/or the heterogeneity of the material.
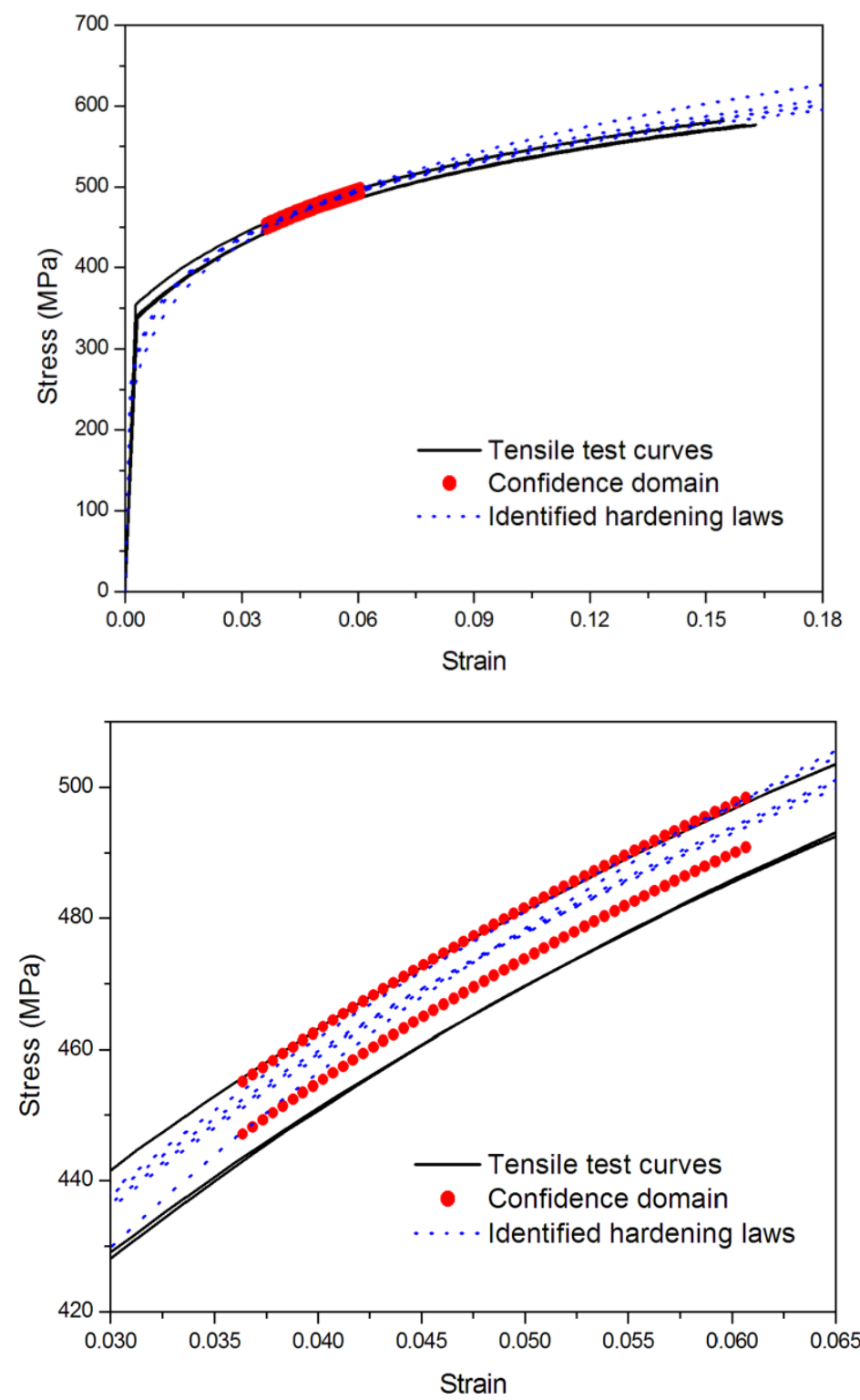

Fig. 15: Comparison between the tensile test curves, the identified hardening laws using the four experimental indentation curves and the confidence domain. (a) Total stressstrain curve (b) zoom around the average representative strain value.

\subsection{Discussion}


As presented in this study, a unique material parameter set $\left(\sigma_{y}, n\right)$ is identified when using one single $F$ - $h$ curve of a spherical indentation test. This result is in agreement with those obtained in multiple studies $[10,12,13,29-31]$. In practice, it is impossible to obtain two or more perfectly similar indentation curves. For the studied case, although the experimental curves are very similar (Fig. 3), the identified parameter sets (Table 3) are different. This result demonstrates the high sensitivity of the instrumented indentation test to the material parameter sets $\left(\sigma_{y}, n\right)$. Therefore, for an experimental case one cannot talk about uniqueness of solution if two or more indentation curves are used. The solution must be a domain that includes the identified parameter sets obtained from each indentation curve. For this reason, the confidence domain was proposed in this study. The proposed confidence domain is an original tool which delimits the identified hardening laws obtained from each experimental indentation curve.

\section{8-Conclusion}

This study presents an investigation of the domain in which the solution exists while identifying the hardening law of a material with spherical indentation using the $F-h$ curve. The $E_{R M S}$ distribution between a reference curve (experimental curve) and a number of $\mathrm{FE}$ simulation curves was studied. This study presents original results and observations that can be summarized as follows:

- The proposed identification method is based on the equation of the error distribution between one experimental $F-h$ curve and FE simulation curves. This method is based on no assumption and gives the material parameter set $\left(\sigma_{y}, n\right)$ that leads to the closest $F-h$ curve to the experimental one.

-The average representative strain indicates the part of the hardening law that is the most precisely identified with the proposed method. All the representative strains in the literature were proposed as an intermediate parameter in the identification process. The use of these representative strains serve as a mathematical trick having no physical basis. In consequence, they are all dependent of human choices and are not strictly determined from the material response. In this study, the identification of material parameter set $\left(\sigma_{y}, n\right)$ is not based on the use of a representative strain. The proposed average representative strain is strictly determined from the material response and is only used for the determination of the confidence domain which takes into account the difference between experimental indentation curves.

-The uniqueness of the solution has been greatly discussed in many studies for the case of spherical indentation. As presented in this study, a unique material parameter set $\left(\sigma_{y}, n\right)$ is identified when using a single $F-h$ curve of a spherical indentation test. However, for the experimental cases, the experimental imprecision and the material heterogeneity lead to different indentation curves, which makes the uniqueness of solution impossible. The proposed solution and confidence domains include all the hardening laws that are solutions for an experimental case. According to the knowledge of the authors, such robust technique does not exist yet in the literature.

\section{References}

[1] Tabor D (1951). Clarendon Press, Oxford

[2] Dao M, Chollacoop N, Van Vliet KJ, Venkatesh TA, Suresh S (2001) Computational modeling of the forward and reverse problems in instrumented sharp indentation. Acta Mater. 49:3899-3918.

[3] Antunes JM, Fernandes JV, Menezes LF, Chaparro BM (2007) A new approach for reverse analyses in depth-sensing indentation using numerical simulation. Acta Mater. 55:69-81. 
[4] Ogasawara N, Chiba N, Chen X. Representative strain of indentation analysis. J. Mater. Res. 2005; 20:2225-2234

[5] Bucaille JL, Stauss S, Felder E, Michler J (2003) Determination of plastic properties of metals by instrumented indentation using different sharp indenters. Acta Mater. 51:1663-1678

[6] Giannakopoulos AE, Suresh S (1999). Determination of elastoplastic properties by instrumented sharp indentation. Scripta Mater 40:1191-1198

[7] Venkatesh TA, Van Vliet KJ, Giannakopoulos AE, Suresh S (2000) Determination of elasto-plastic properties by instrumented sharp indentation: guidelines for property extraction. Scripta mater. 42:833-839

[8] Taljat B, Zacharia T, Kosel F (1998) New analytical procedure to determine stress-strain curve from spherical indentation data. Int. J. Solids Struct. 33:4411-4426.

[9] Lee H, Lee J, Pharr GM (2005) A numerical approach to spherical indentation techniques for material property evaluation. J. Mech. Phys. Solids. 53:2037-2069.

[10] Cao YP, Lu J (2004) A new method to extract the plastic properties of metal materials from an instrumented spherical indentation loading curve. Acta Mater. 52:4023-4032

[11] Zhao M, Ogasawara N, Chiba N, Chen X (2006) A new approach to measure the elastic-plastic properties of bulk materials using spherical indentation. Acta Mater. 54:23-32

[12] Cao Y, Qian X, Huber N (2007) Spherical indentation into elastoplastic materials: Indentationresponse based definitions of the representative strain. Mater. Sci. Eng. A. 454-455:1-13

[13] Lee JH, Kim T, Lee H (2010) A study on robust indentation techniques to evaluate elastic-plastic properties of metals. Int. J. Solids Struct. 2047:647-664

[14] Ogasawara N, Chiba N, Chen X (2009) A simple framework of spherical indentation for measuring elastoplastic properties. Mech. Mater. 41:1025-1033

[15] Ahn JH, Kwon D (2001) Derivation of plastic stress-strain relationship from ball indentations: Examination of strain definition and pileup effect. J. Mater. Res. 16:31703178

[16] Jeon EC, Baik MK, Kim SH, Lee BW, Kwon D (2005) Determining Representative Stress and Representative Strain in Deriving Indentation Flow Curves Based on Finite Element Analysis. Eng. Mater. 294-300:2152-2157

[17] Jeon EC, Kim JY, Baik MK, Kim SH, Park JS, Kwon D (2006) Optimum definition of true strain beneath a spherical indenter for deriving indentation flow curves. Mater. Sci. Eng. A. 419:196-201

[18] Chaudhri MM (1998) Subsurface strain distribution around Vickers hardness indentations in annealed polycrystalline copper. Acta Mater. 46:3047-3056

[19] Chaudhri MM (2000) Strain hardening around spherical indentations. Phys. Stat. Sol. 182:641652

[20] Collin JM, Parenteau T, Mauvoisin G, Pilvin P (2009) Material parameters identification using experimental continuous spherical indentation for cyclic hardening Comp. Mater. Sci. 46:333-338

[21] Moussa C, Bartier O, Mauvoisin G, Pilvin P, Delattre G (2012) Characterization of homogenous and plastically graded materials with spherical indentation and inverse analysis. J. Mater. Res. 27:2027

[22] Chaudhri MM (1996) Subsurface plastic strain distribution around spherical indentations in metals. Philos.1 Mag. A. 74: 1213-1224

[23] Meyer E (1908) Untersuchungen über Härteprüfung und Härte. Phys. Z. 9: 66-74

[24] Prou J, Kishimoto K, Constantinescu A (2010) Identification of Young's Modulus from indentation testing and inverse analysis. J. Solids Mech. Mater. Eng. 4:781-795 
[25] Gailletaud G, Pilvin P (1993) Winter Annual Meeting Symposium on Material Parameter Estimation for Modern Constitutive Equations, USA

[26] Isselin J, Iost A, Golek J, Najjar D, Bigerelle M (2006) Assessment of the constitutive law by inverse methodology: Small punch test and hardness. J. Nucl. Mater. 352:97-106

[27] Cheng YT, Cheng CM (2004) Scaling, dimensional analysis, and indentation measurements. Mater. Sci. Eng. 44:91-149

[28] Cheng YT, Cheng CM (1999) Can stress-strain relationships be obtained from indentation curves using conical and pyramidal indenters? J. Mater. Res. 14:3493-3496

[29] Lan H, Venkatesh TA (2007) On the uniqueness and sensitivity issues in determining the elastic and plastic properties of power-law hardening materials through sharp and spherical indentation Philos. Mag.. 87:4671-4729

[30] Chen X, Ogasawara N, Zhao M, Chiba N (2007) On the uniqueness of measuring elastoplastic properties from indentation: The indistinguishable mystical materials. J. Mech. Solids. 55:1618-1660

[31] Collin JM, Mauvoisin G, Pilvin P, El Abdi R (2008) Use of spherical indentation data changes to materials characterization based on a new multiple cyclic loading protocol. Mater. Sci. Eng. A. 488: 608-622. 